\title{
Recent Developments in Gravitational Microlensing and the Latest MACHO Results: Microlensing Towards the Galactic Bulge
}

\author{
D.P. Bennett ${ }^{\dagger, *}$, C. Alcock ${ }^{*, \dagger}$, R.A. Allsman*, \\ T.S. Axelrod*, K.H. Cook ${ }^{*}$, , K.C. Freeman ${ }^{\ddagger}$, \\ K. Griest ${ }^{\dagger}, \|$, S.L. Marshall ${ }^{\dagger, b}$, S. Perlmutter ${ }^{\dagger}$, \\ B.A. Peterson ${ }^{\ddagger}$, M.R. Pratt $t^{\dagger, b}$, P.J. Quinn ${ }^{\ddagger}$, A.W. Rodgers ${ }^{\ddagger}$, \\ C.W. Stubbs ${ }^{\dagger, \star}$, W. Sutherland \\ (The MACHO Collaboration) \\ * Lawrence Livermore National Laboratory, Livermore, CA 94550 \\ ${ }^{\dagger}$ Center for Particle Astrophysics, University of California, \\ Berkeley, CA 94720 \\ $\ddagger$ Mt. Stromlo and Siding Spring Observatories, \\ Australian National University, Weston, ACT 2611, Australia \\ \| Department of Physics, University of California, San Diego, CA 92039 \\ b Department of Physics, University of California, Santa Barbara, CA 93106 \\ * Department of Astronomy, University of Washington, Seattle, WA 98195 \\ - Department of Physics, University of Oxford, Oxford OX1 3RH, U.K.
}

\begin{abstract}
We review recent gravitational microlensing results from the EROS, MACHO, and OGLE collaborations, and present some details of the very latest MA$\mathrm{CHO}$ results toward the Galactic Bulge. The MACHO collaboration has now discovered in excess of 40 microlensing events toward the Galactic Bulge during the 1993 observing season. A preliminary analysis of this data suggests a much higher microlensing optical depth than predicted by standard galactic models suggesting that these models will have to be revised. This may have important implications for the structure of the Galaxy and its dark halo. Also shown are MACHO data of the first microlensing event ever detected substantially before peak amplification, the first detection of parallax effects in a microlensing event, and the first caustic crossing to be resolved in a microlensing event.
\end{abstract}

\section{Introduction}

The suggestion by Paczyński (1986) that compact objects in the halo of our Galaxy might be detected by means of gravitational microlensing was confirmed in a rather spectacular fashion last September when the EROS, MACHO, and OGLE collaborations each discovered their first candidate microlensing events in the course of less than a month (Alcock, et al., 1993, Aubourg, et al., 1993, and Udalski, et al., 1993). Since then, the total number of microlensing events 
discovered has grown to about 60, and the implications of the microlensing results toward both the Large Magellanic Cloud and the Galactic bulge remain somewhat mysterious.

The basic physics of microlensing is quite simple. If a compact object passes very close to the line of sight to a background star, the light will be deflected to produce two images of the star. In the case of perfect alignment, the star will appear as an 'Einstein ring' with a radius of

$$
r_{E}=\sqrt{\frac{4 G M L x(1-x)}{c^{2}}},
$$

where $M$ is the lens mass, $L$ is the observer-source distance and $x$ is the ratio of the observer-lens and observer-source distances. In a typical situation of imperfect alignment, the image appears as two arcs. On cosmological scales, many cases of quasars multiply imaged by foreground galaxies are known ; however, for stellar lensing the angular separation of the lensed images is much smaller. For a source distance of $50 \mathrm{kpc} \approx 10^{10} \mathrm{AU}$ and a lens distance of $10 \mathrm{kpc}$, the Einstein radius is $r_{E} \approx 8 \sqrt{M / \mathrm{M}_{\odot}} \mathrm{AU}$; this yields an angular separation of $\sim 0.001$ arcsecond which is well below even the Space Telescope resolution, hence the term 'microlensing'.

However, in the point source approximation, the lensing produces a net amplification of the source by a factor

$$
A=\frac{u^{2}+2}{u \sqrt{u^{2}+4}}
$$

where $u=b / r_{E}$ and $b$ is the distance of the lens from the direct line of sight. (The above scale of $8 \mathrm{AU}$ is much larger than the radius of a star, hence the point source approximation is accurate for lens masses $\left.\gtrsim 10^{-5} \mathrm{M}_{\odot}\right)$. The amplification is approximately $u^{-1}$ for $u \lesssim 0.5$, and $1+2 u^{-4}$ for $u \gg 1$, hence the amplification can be very large but falls rapidly for $u \gtrsim 1$. Since objects in the Galaxy are in relative motion, this amplification will be time-dependent; for a typical lens transverse velocity of $200 \mathrm{~km} / \mathrm{s}$, the duration is

$$
\hat{t} \equiv \frac{2 r_{E}}{v_{\perp}} \approx 140 \sqrt{M / \mathrm{M}_{\odot}} \text { days. }
$$

This is a convenient timescale for astronomical observations, and thus by sampling on a range of timescales the microlensing searches may be sensitive to a wide mass range from small planets of $\sim 10^{-6} \mathrm{M}_{\odot}$ to black holes of $\sim 100 \mathrm{M}_{\odot}$, covering most of the plausible candidates.

\section{Optical Depth}

The 'optical depth' $\tau$ for gravitational microlensing is defined as the probability that a given star is lensed with $u<1$ or $A>1.34$ at any given time, and is

$$
\tau=\pi \int_{0}^{L} \frac{\rho(l)}{M} r_{E}^{2}(l) d l
$$


where $l$ is the distance along the line-of-sight and $\rho$ is the dark matter density. Since $r_{E} \propto \sqrt{M}$, while for a given $\rho$ the number density of lenses $\propto M^{-1}$, the optical depth is independent of the individual MACHO masses. Using the virial theorem, it is found that $\tau \sim(v / c)^{2}$, where $v$ is the rotation speed of the Galaxy. More detailed calculations (Griest, 1991) give an optical depth for lensing by halo dark matter of stars in the Large Magellanic Cloud (LMC) of

$$
\tau_{\mathrm{LMC}} \approx 5 \times 10^{-7}
$$

This very low value is the main difficulty of the experiment; only one star in two million will be amplified by $A>1.34$ at any given time, while the fraction of variable stars is much higher, $\sim 3 \times 10^{-3}$.

\section{Microlensing Signatures}

Fortunately, microlensing has many strong signatures which can discriminate it from stellar variability :

1) Since the optical depth is so low, only one event should be seen in any given star.

2) The deflection of light is wavelength-independent, hence the star should not change color during the amplification.

3) The accelerations of galactic objects are negligible on timescales of these events, hence the events should be symmetrical in time, and have a shape derived from $(2)$ with $u(t)=\sqrt{u_{\min }^{2}+\left(v_{\perp}\left(t-t_{\max }\right) / r_{E}\right)^{2}}$. Examples of such light-curves are shown in Figure 2.

All these characteristics are distinct from known types of intrinsic variable stars; most variable stars are periodic or semi-regular, and do not remain constant for long durations. They usually change temperature and hence color as they vary, and they usually have asymmetrical lightcurves with a rapid rise and slower fall.

In addition to these individual criteria, if many candidate microlensing events are detected, there are further statistical tests that can be applied:

4) The events should occur equally in stars of different colors and luminosities.

5) The distribution of impact parameter $u_{\min }$ should be uniform from 0 to the experimental cutoff $u\left(A_{\min }\right)$.

6) The event timescales and peak amplifications should be uncorrelated. (In practice these distributions will be modulated by the detection efficiencies, which can be computed from simulations).

It is worth clarifying two common misconceptions: first, this experiment does not provide an estimate of $\Omega$, since the amount of dark matter within 50 kpc of spiral galaxies is approximately known from rotation curve data. This represents $\Omega \sim 0.05$ : if $\Omega=1$ either the halos must extend far beyond $50 \mathrm{kpc}$ or there must be intergalactic dark matter. Secondly, the lenses are not required to be dark, merely significantly fainter than the source stars. However, since the LMC is located $30^{\circ}$ from the galactic plane, the optical depth from known stars is only $\lesssim 10^{-8}$ (Bahcall, et al., 1994).

\section{Microlensing Searches}

Due to the low optical depth, a very large number of stars must be monitored over a long period to achieve a significant detection rate. The optimal 
targets when searching for Machos in the galactic halo are the Large and Small Magellanic Clouds, the largest of the Milky Way's satellite galaxies, since they have a high surface density of stars and are distant enough at 50 and $60 \mathrm{kpc}$ to provide a good path length through the dark halo. This requires a Southern hemisphere observatory.

Another target for microlensing seaches is the Galactic bulge where a higher microlensing optical depth is predicted due to lensing by faint stars in the Galactic disk (Griest, et al., 1991, Paczyński, 1991). Naively, one might expect that microlensing toward the bulge would not be of great relevance for the halo dark matter problem, but as we shall see, this is not the case.

There are curently three microlensing surveys that are taking data and have reported results: the EROS, MACHO, and OGLE collaborations. The EROS collaboration operates two seperate experiments which observe the LMC. They search for events lasting from days to months using photographic plates taken at the ESO Schmidt and for events lasting less than a day from a $40-\mathrm{cm}$ telescope with a large format CCD array camera also located at the ESO site in La Silla, Chile. They plan to upgrade to a dedicated 1-m telescope with a very large format two color CCD camera system (64 or 128 Mega-pixels) in late 1995 or 1996. The OGLE collaboration now observes the Galactic bulge with $\sim 75$ nights per year on the 1-m Swope telescope at Las Campanas, Chile, but they also plan to upgrade to a dedicated 1.3-m telescope in 1995 or 1996.

The MACHO collaboration has full-time use of the $1.27-\mathrm{m}$ telescope at Mt.Stromlo Observatory near Canberra, Australia, which is used to image 0.5 square degrees of sky simultaneously in two color bands. The two foci are equipped with large CCD cameras (Stubbs, et al., 1993), each containing 4 Loral CCD chips of $2048 \times 2048$ pixels. Most of the observing time is divided between the LMC and bulge, with a small amount of time being spent on the SMC.

\section{Microlensing Towards the LMC}

The first Galactic microlensing events every discovered were announced simultaneously by the EROS and MACHO collaboration in the fall of 1993 (Alcock, et al., 1993, Aubourg, et al., 1993). These data initiated a flurry of speculation as to whether the the halo might be entirely baryonic or whether some non-halo population might be responsible for the microlensing toward the LMC. Although the EROS and MACHO collaborations have not yet announced definitive results on the microlensing optical depth toward the LMC, preliminary results given in a number of conferences suggest that the optical depth is both significantly below the prediction $\left(\tau \approx 5 \times 10^{-7}\right)$ for a "standard halo" composed entirely of Machos and significantly above the maximum possible value $(\tau<$ $10^{-8}$ ) due to known distributions of stars (Bahcall et al., 1994). The possibility that these events are not microlensing but rather a new variable star phenomena now seems rather unlikely as spectral follow-up has confirmed that they appear to be normal stars (Della Valle, 1994, Beaulieu, et al., 1994, Giraud, 1994, and Alcock, et al., 1994a).

One possibility that remains viable is that the microlensing may be due to faint stars in the LMC (Sahu, 1994), but this seems unlikely for several reasons. First, to get the required event rate, one need to assume that the mass of the LMC is almost entirely baryonic while the Milky Way is apparently dominated by non-baryonic matter. It is difficult to see how this could come 
about. Also, although microlensing by a self-consistent model of the LMC which is massive enough to explain the observed events has never been investigated, naive arguments suggest that the timescales of the observed events could most easily be explained by microlensing by objects of substellar mass.

Thus, the most reasonable conclusion is that the microlensing is due to brown dwarfs in a halo or perhaps spheroidal population (Giudice, Mollerach, \& Roulet, 1993), but that the these objects do not provide the dominant mass for a "standard halo". It is tempting to conclude that there must be another component that dominates the halo, except that, as we shall see, the microlensing optical depth toward the galactic bulge is much higher than expected. Thus, another viable possibility is that the dynamics of the inner galaxy are dominated by a massive disk and bulge, and that Machos are the dominant component of a much smaller than "standard" halo.

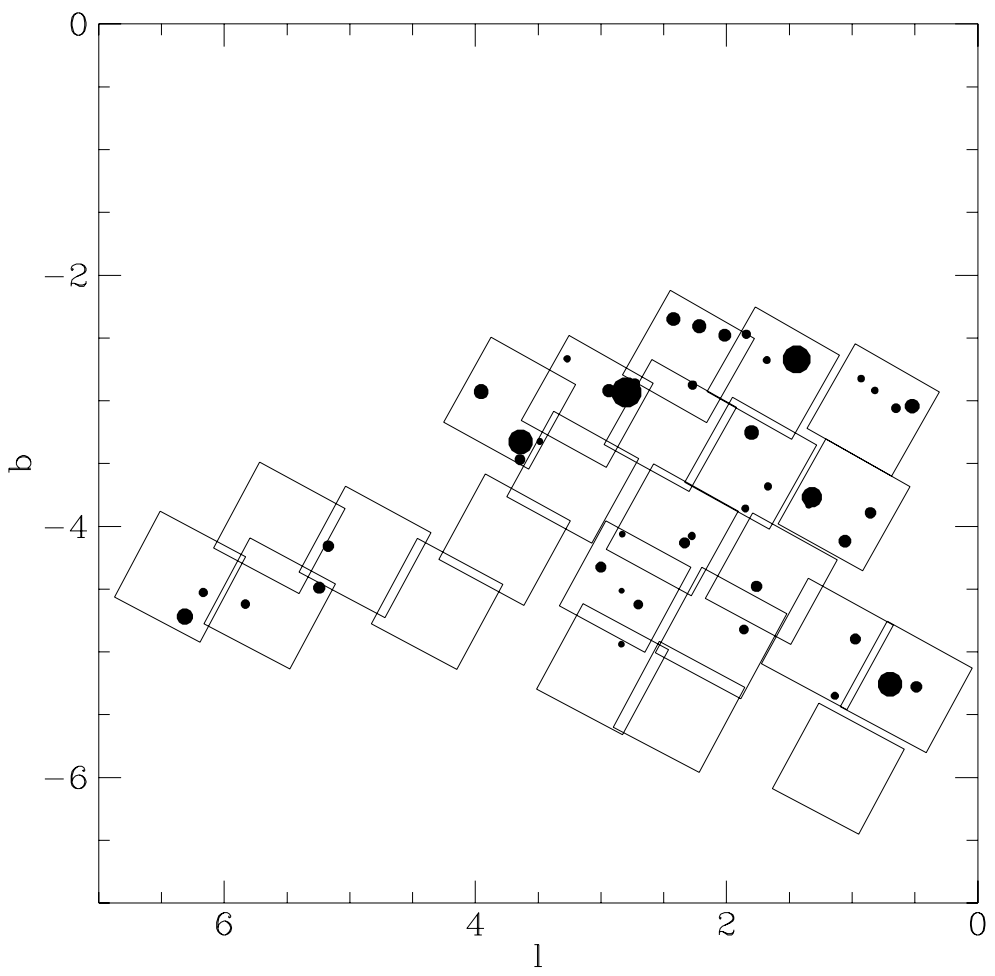

Figure 1. The location of the 24 bulge fields that were observed by the MACHO group and analyzed for this paper. The spots indicate the locations of detected events, and the spot area is proportional to the $\hat{t}$ value for the event.

\section{Microlensing Events Toward the Galactic Bulge}

Within a week after the EROS and MACHO teams announced the first microlensing events detected toward the LMC, the OGLE collaboration had discovered the first microlensing event ever seen toward the Galactic bulge (Udalski, et al., 1993). Subsequent analyses by the OGLE group revealed about 10 additional events, and revealed a very high optical depth towards Baade's window (Udalski, et al., 1994a). These results are fully consistent with the MACHO 
results that will be discussed in the remainder of this paper.

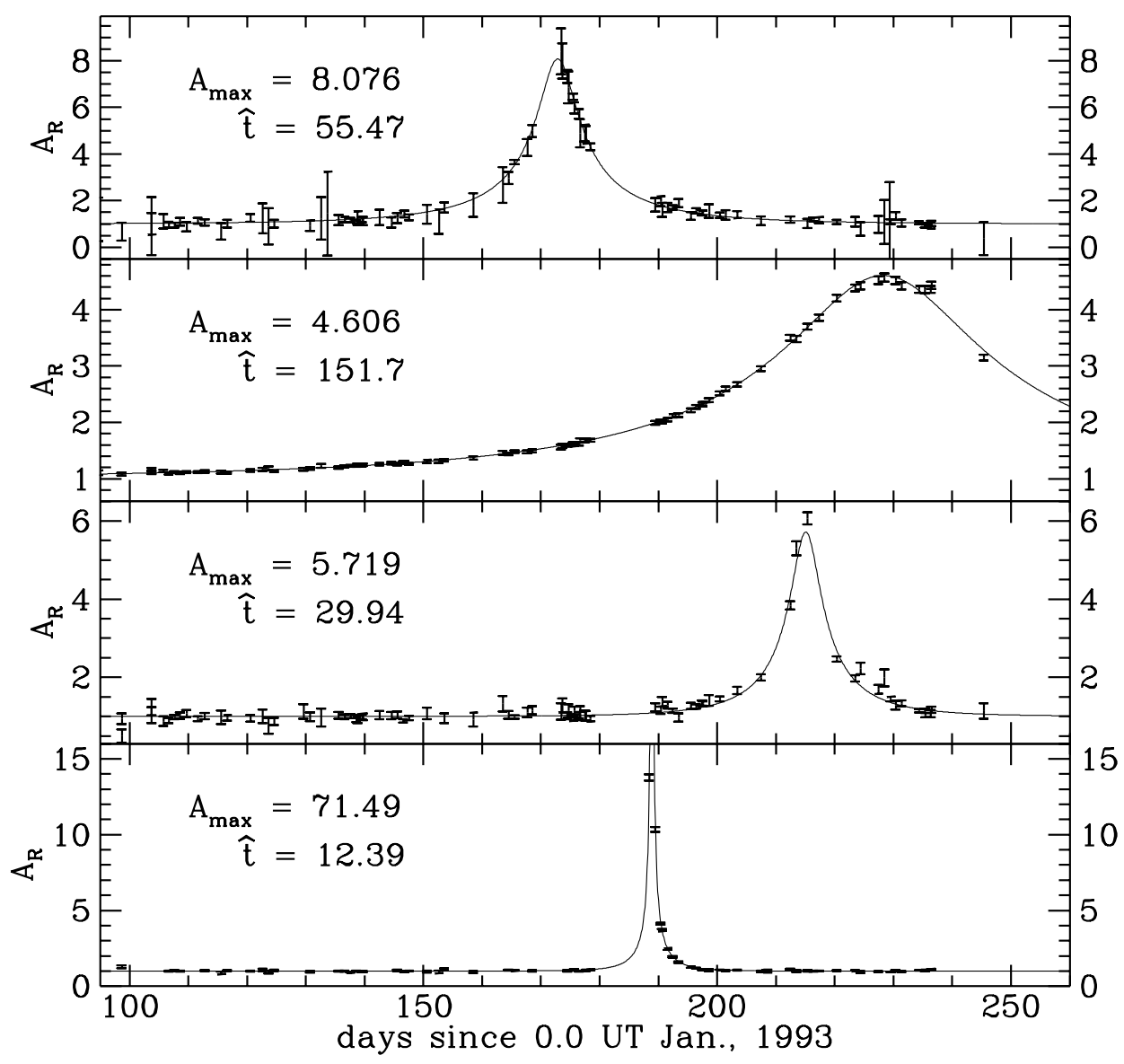

Figure 2. MACHO-Red band lightcurves of 4 events from field 101 located at $\ell \approx 3.8, b \approx-3.1$.

We have recently analyzed the 2-color light curve data for about 10 million in 24 fields toward the Galactic bulge. The location of these fields in galactic coordinates (as well as the location of the events) in shown in Fig. 1. Before starting the microlensing search, measurements with questionable PSF chi square, crowding, missing pixel, or cosmic ray flags are flagged as bad measurements and removed from further consideration. Then, we run matched filters through the data in both colors, and 37,000 stars passed the filter trigger threshold and were fit jointly in both colors with a microlensing light curve. These stars form our "level 1" candidate list and are subject to further cuts on the difference between the $\chi^{2}$ values for the microlensing and flux=const. fit, and the microlensing fit $\chi^{2}$ per d.o.f. Further cuts are made on the number of data points near the peak and the average estimated error. Four of the 45 candidates which pass these cuts are shown in Fig. 2. A few of the 45 events may be variable stars, but the majority are almost certainly microlensing events. A color magnitude diagram showing the locations of these 45 stars as well as $7 \%$ of the stars within 2.5' of these stars is shown in Fig. 3. 


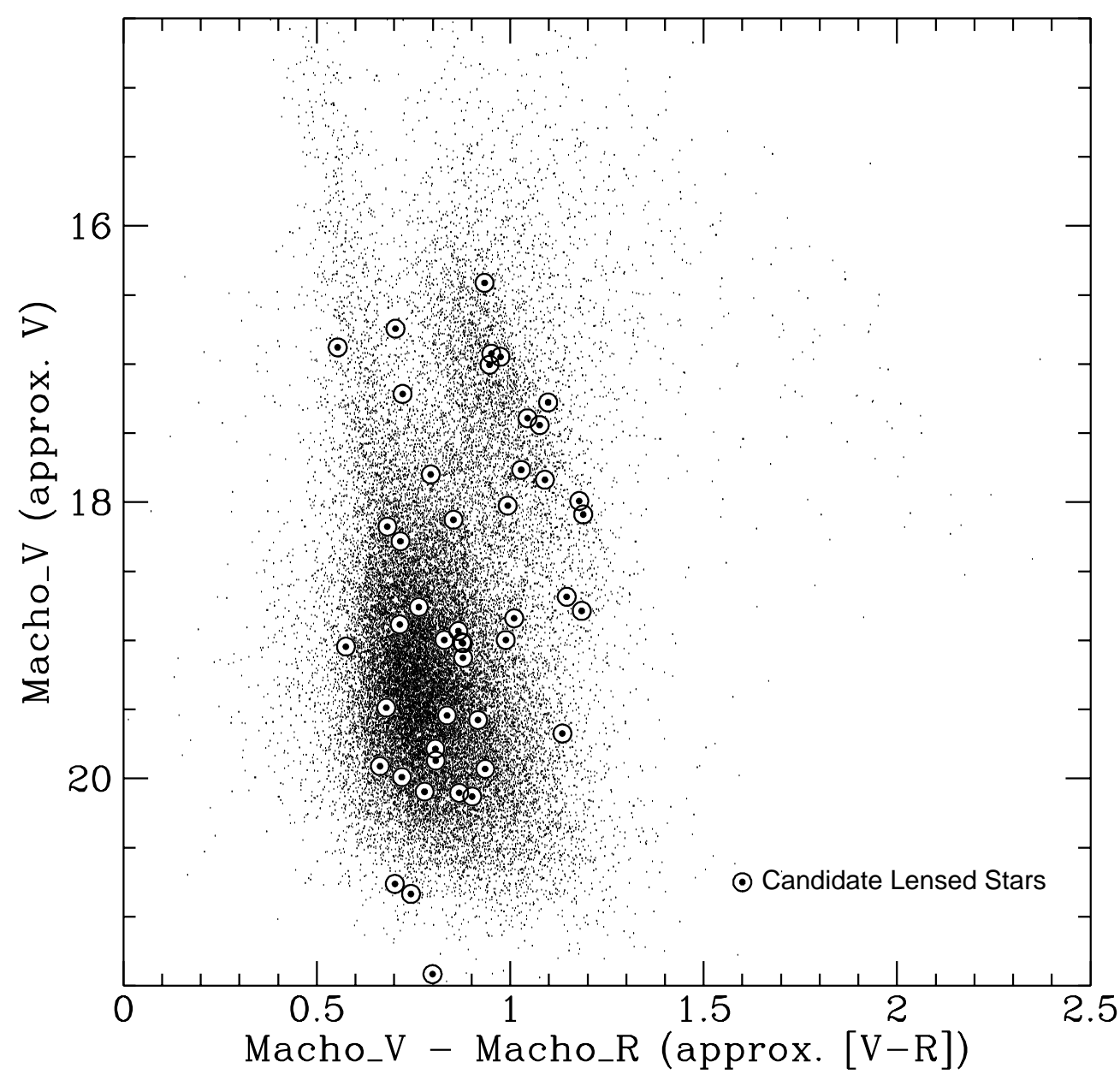

Figure 3. A color magnitude diagram for the 45 microlensing candidates and $7 \%$ of the stars that fall within 2.5 ' of the candidates.

\section{Preliminary Optical Depth Estimates}

The primary measurable quantity in a gravitational microlensing experiment is the microlensing optical depth which is defined to be the instantaneous probability that a random star is magnified by a lensing object by more than a factor of 1.34. This is a measure of the mass in microlensing objects along the line of sight to the source stars. Experimentally, one can define the measured optical depth as the amount of time that the microlensed stars spend inside the Einstein ring (i.e.are amplified by more than 1.34).

$$
\tau_{\text {meas }}=\frac{1}{E} \frac{\pi}{4} \sum_{i} \frac{\hat{t}_{i}}{\epsilon\left(\hat{t}_{i}\right)} .
$$

where $E$ is the total exposure (in star-years), $\hat{t}_{i}$ is the Einstein ring diameter crossing time, and $\epsilon\left(\hat{t}_{i}\right)$ is the detection efficiency. 
The detection efficiency $\epsilon$ is properly determined by adding "fake" stars with the same luminosity function as the real stars to the raw images. The brightnesses of these "fake" stars can be modulated according to randomly selected microlensing light curves, and then the analysis can be run to see how many of these simulated events are recovered. A much simpler procedure would be to add simulated microlensing light curves to the light curve database, but this yields $\epsilon$ values that are generally too large because it neglects the effects of systematic errors in the photometry and the blending of stellar images which tends to make lensing events more difficult to see. The $\epsilon$ values calculated in this way are known as sampling efficiencies, and these are the only efficiencies that we will present here. $\epsilon(\hat{t})$ is plotted for several different data cuts in Fig. 4.

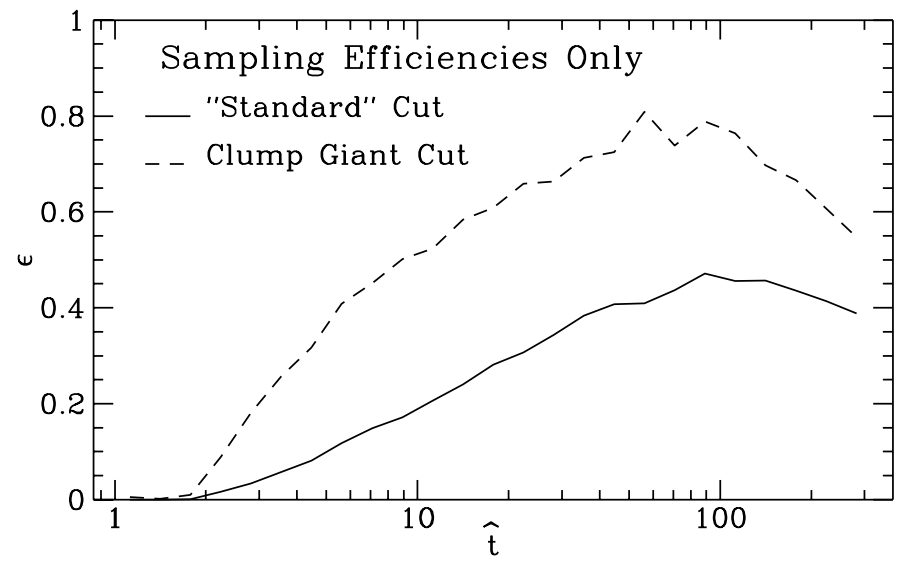

Figure 4. Sampling Efficiencies are plotted for our standard and clump giant cuts. The efficiencies are higher for clump giants because they are much brighter than the average star.

\section{Microlensing Optical Depth Towards the Galactic Bulge $\times 10^{6}$}

\begin{tabular}{|c|c|c|c|c|c|c|c|c|}
\hline \multicolumn{7}{|c|}{} & \multicolumn{7}{|c|}{ Confidence Levels } \\
\hline cut & $\mathrm{N}$ & 0.025 & 0.05 & 0.16 & measured & 0.84 & 0.95 & 0.975 \\
\hline standard & 43 & 1.02 & 1.11 & 1.29 & $\mathbf{1 . 5 8}$ & 1.93 & 2.16 & 2.28 \\
clump giant & 13 & 1.25 & 1.48 & 2.06 & $\mathbf{3 . 0 3}$ & 4.47 & 5.46 & 5.96 \\
$|b|<3.1$ & 18 & 1.81 & 2.11 & 2.72 & $\mathbf{3 . 7 9}$ & 5.27 & 6.26 & 6.83 \\
clump giant & 9 & 2.04 & 2.57 & 3.91 & $\mathbf{6 . 3 2}$ & 10.27 & 13.07 & 14.48 \\
w $/|b|<3.1$ & & & & & & & & \\
\hline
\end{tabular}

Table 1. The opticcal depth $\tau$ is listed in units of $10^{-6}$ for the different cuts discussed in the text. Note that the $|b|<3.1$ cuts have selection effects that have not been included in the confidence level estimation.

Another problem that interferes with the determination of the microlensing optical depth toward the Galactic Bulge is that many of the stars that we see 
when we look towards the galactic bulge are foreground disk stars. If we do not correct for this, then our estimates of the optical depth $\tau$ will be underestimates. One way to avoid both the problem of foreground stars and the complications due to stellar blends is to concentrate on a class of stars that is both bright and "known" to be in the galactic bulge: the "clump giant" stars. These are relatively low mass core helium burning giants-the horizontal branch of a metal rich population. Their location is marked on the color magnitude diagram in Fig. 3.

The measured optical depths for a number of different cuts on the data are shown in Table 1. This table displays the measured optical depth and various confidence level limits on the microlensing optical depth for several different cuts on the bulge data sample. The optical depth $\tau$ corresponding to a confidence level, $P$, is the $\tau$ value such that a fraction $P$ of simulated data sets have a measured optical depth $>\tau$. The simulated data sets are constructed assuming Poisson statistics and an actual $\hat{t}$ distribution that is identical to the observed $\hat{t}$ distribution.

The standard cut referred to in Table 1 is quite similar to the one which generated the 45 events shown in Fig. 2, but a few more cuts had to be added for consistency with the monte carlo events used for the efficiency estimation. Two additional types of cuts are referred to in Table 1. The first is the clump giant cut alluded to above. The fact that this cut seems to imply a noticeably higher optical depth $\left(\tau=3.0_{-0.9}^{+1.5} \times 10^{-6}\right)$ than the standard cut $\left(\tau=1.58_{-0.28}^{+0.35} \times\right.$ $10^{-6}$ ) suggests that the full efficiency corrections and/or the foreground star corrections are important for the full sample.

The other cut referred to in Table 1 is a cut to include only the 5 fields which have a central galactic latitude of $|b|<3.1$. This cut is intended to emphasize the contribution of microlensing by objects in the galactic disk as opposed to the galactic bulge or bar. A galactic bar elongated along the line of sight has been proposed as the source of the large microlensing optical depth seen toward the galactic bulge by the OGLE group (Paczyński, et al., 1994) and by Zhou, Spergel, and Rich (1994). We (Alcock, et.al., 1994b) and Gould (1994b) have suggested that a heavy, "maximal" disk might account for the large optical depth seen toward the bulge. The results shown in Table 1 do seem to suggest that microlensing by the disk is contributing a large fraction of the total optical depth, but we should caution that the cut at $|b|<3.1$ has been selected after the distribution of events with $b$ was known. So, this cut can be considered to have been made in a biased way. A more sophisticated analysis is now under way to determine if, in fact, these data indicate that the "maximal disk" model is preferred.

\section{An "Alert" Event}

Fig. 5 shows the light curve of the first gravitational microlensing event ever detected substantially before its peak amplification. The time of discovery is indicated. An IAU circular (IAUC 6068) was submitted about 24 hours after the event was discovered, and a great deal of photometric and spectroscopic data was collected during the course of the event. The team of Benetti, Pasquini, West, and de Lapparent obtained spectra on 6 different nights from the NTT and $3.6 \mathrm{~m}$ telescopes at ESO, and have confirmed that the spectral features of the star did not change as the star rose to a peak amplification of $A=3.5$ and then dropped back down to its usual brightness (IAUC 6069 and 6071). Similar data 
was obtained at a number of large telescopes including a spectrum obtained by Dick Hunstead at the AAT on Sept. 2. The ESO and AAT data indicate that star is classified as a K0 class II-IV star with a radial velocity of $\sim-200 \mathrm{~km} / \mathrm{sec}$.

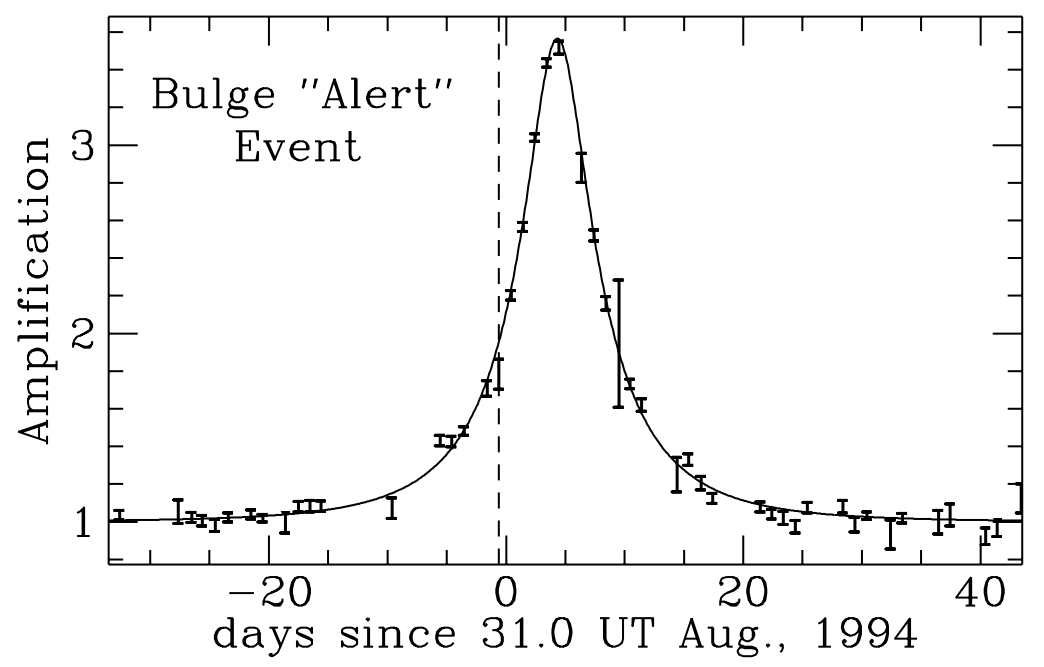

Figure 5. The light curve of the first microlensing event discovered substantially before peak amplification. The vertical dashed line is the time of discovery.

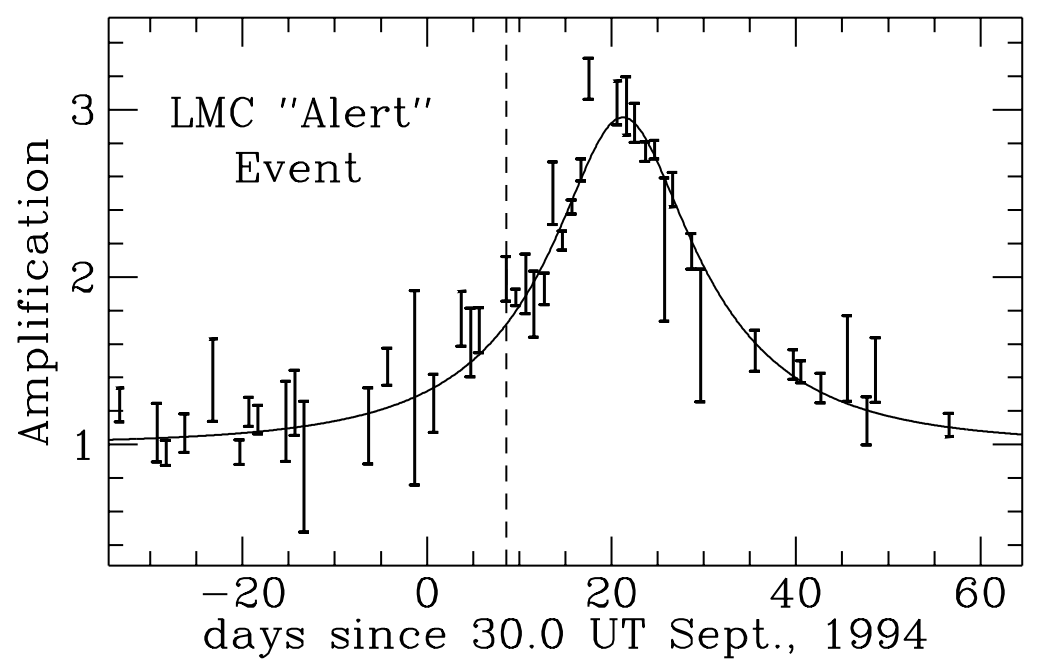

Figure 6. The light curve of the first microlensing event discovered in progress towards the LMC. The vertical dashed line is the time of discovery.

An alert system has also been demonstrated by the OGLE collaboration (Udalski, et.al., 1994c) who have detected two moderate amplification events in progress.

At the time of this conference, the first microlensing event ever detected in real time toward the LMC appears to be in progress!! The light curve of this event is shown in Fig. 6. Giraud (private communication) obtained a spectrum of this star near peak amplifiction, and confirmed that it is a normal $\mathrm{F}$ star. 
A Parallax Event

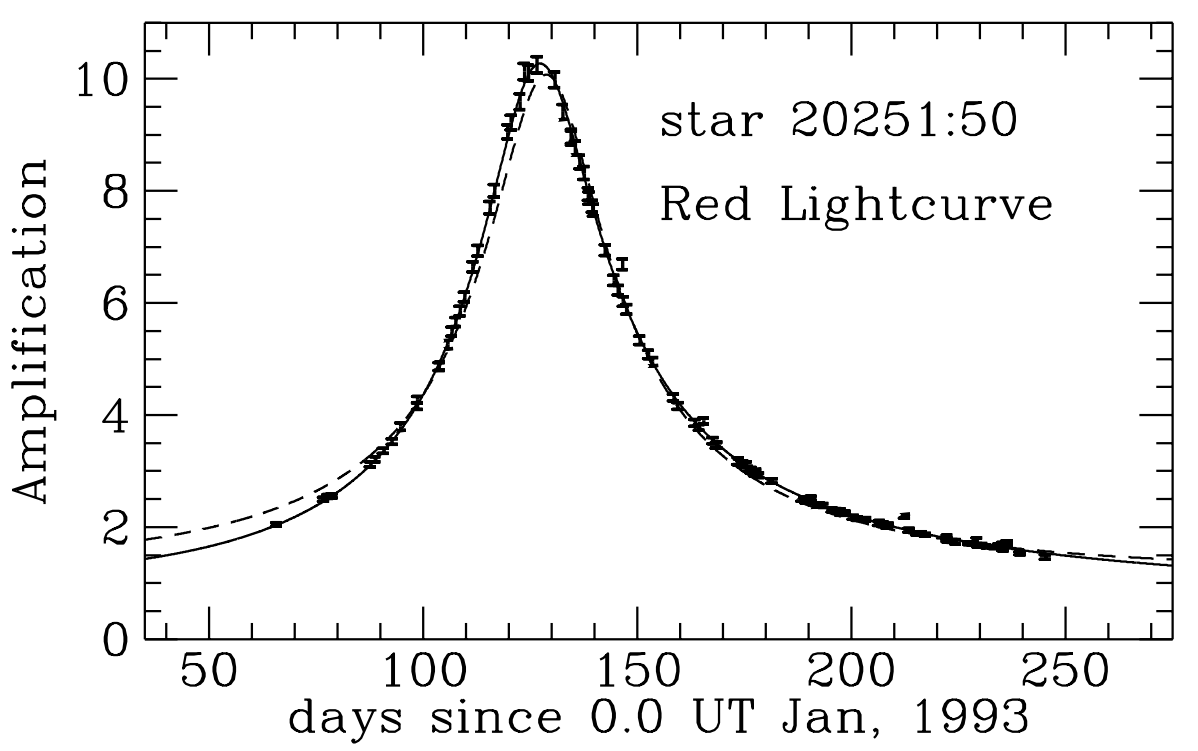

Figure 7. The MACHO-Red band light curve for the longest event yet detected by the MACHO project. The solid curve is the fit light curve which includes the effect of the earth's motion, while the dashed curve is the best fit ignoring the motion of the earth.

Fig. 7 shows the light curve of the longest timescale microlensing event that we have detected, and the first to show a significant asymmetry in it's light curve. This asymmetry can be explained as a parallax effect-the change in the earth's velocity over the hundreds of days that this event has lasted has caused a deviation from the simple 3-parameter light curve that describes most microlensing events. This light curve is well fit by a model which includes the orbital motion of the earth with the transverse velocity of the microlensing object projecting to a transverse velocity of $54 \mathrm{~km} / \mathrm{sec}$ at the position of the earth. This gives us the information we need to determine the mass of the microlensing object as a function of the earth-lens distance. The implied mass is shown in Fig. 8. It seems clear from Fig. 8 that the lensing object must very likely be a low mass star in the Galactic disk or a brown dwarf in the galactic bulge.

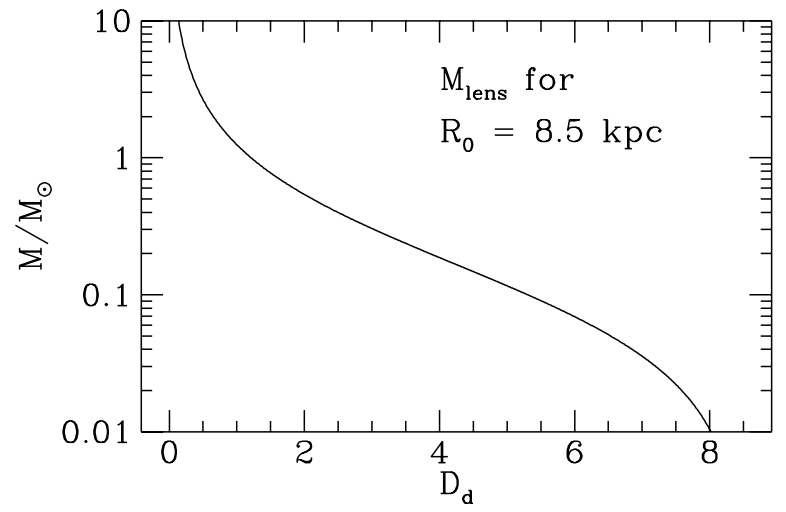

Figure 8. The implied mass is plotted as a function of the distance to the lens 
for the parallax event.

Much progress could be made toward resolving the mysteries of microlensing if the parallax effect could be observed for a large number of microlensing events. However, it is only for the very long events that the earth will move enough during the event for such an effect to be detectable. This situation could be remedied if a small satellite could observe events from a solar orbit, as suggested by Gould (1994a-b). The satellite would need to be alerted to events detected in progress from the ground, but this capability has already been demonstrated by the OGLE and MACHO groups.

MACHO Observations of Microlensing by a Binary

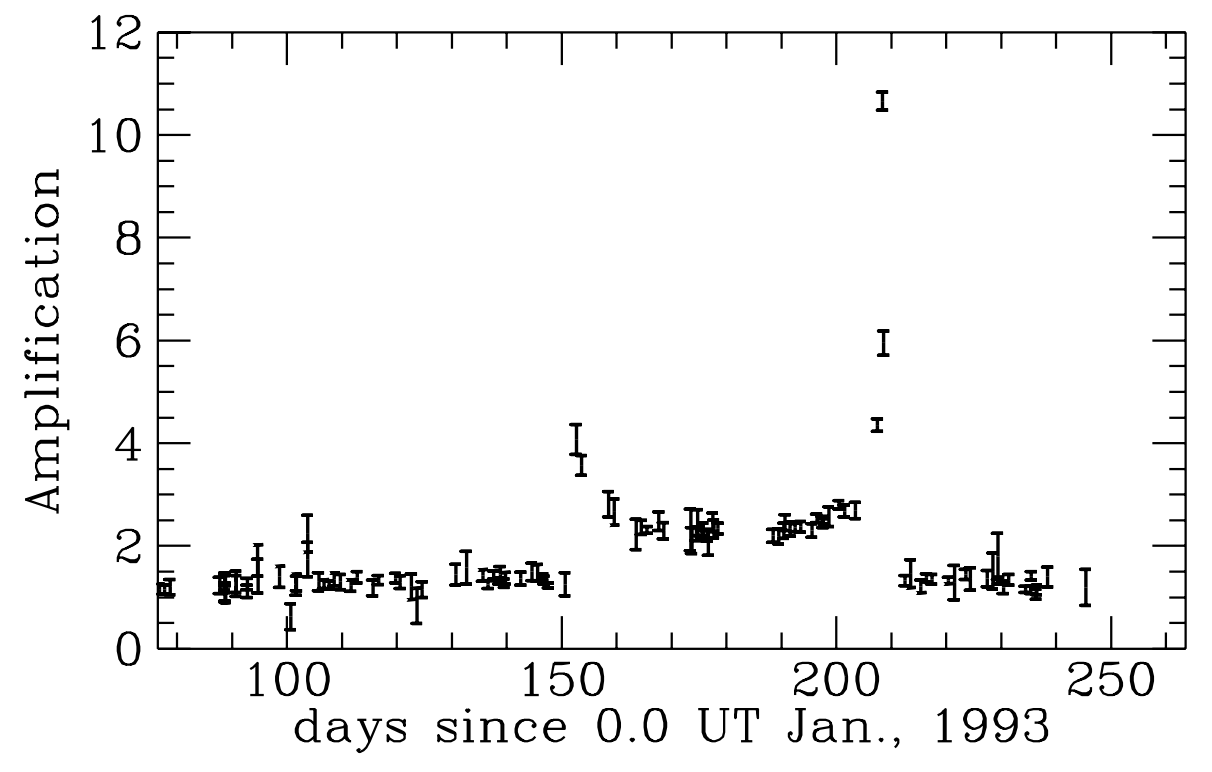

Figure 9. The MACHO-Blue band light curve of the binary lens event first seen by OGLE.

Fig. 9 shows light curves of a microlensing event that clearly shows characteristics of a binary lens with "caustic crossings" where the source star crosses into (or out of) a region where two extra images are created (making a total of 5). This event was first discovered by the OGLE collaboration (Udalski, et.al., 1994c), but the MACHO data provides a nice confirmation with very good coverage of the second caustic crossing which was largely missed by OGLE. In fact, the MACHO coverage of the second caustic crossing is so good, that the finite angular size of the source star is resolved in the MACHO data. The divergent amplification of the 4th and 5th images near a caustic can be shown to have the form $A \approx d^{-1 / 2}$ where $d$ is the distance to the caustic on the interior side. The modification of this formula for a finite size source star with a realistic limb darkening law has been derived by Schneider and Wagoner (1987). Fig. 10 shows a fit using the $4 \mathrm{MACHO}$ Blue band data points closest to the caustic using a 3 parameter model with the Schneider and Wagoner profile. The fit $\chi^{2}=0.009$ for 1 d.o.f. This fit fixes the time for the caustic to cross the diameter of the star 
to be 10 hours. Using the OGLE fit value for the angle of the caustic crossing, we find that the time for the lens position to cross the diameter of the star is about 8 hours. This implies a lens velocity of $v_{t}=x(48 \mathrm{~km} / \mathrm{sec}) R_{\text {star }} / R_{\odot}$, where $x$ is the fractional distance between us and the lensing object. This will lead to a contraint on the mass of and distance to the lens.

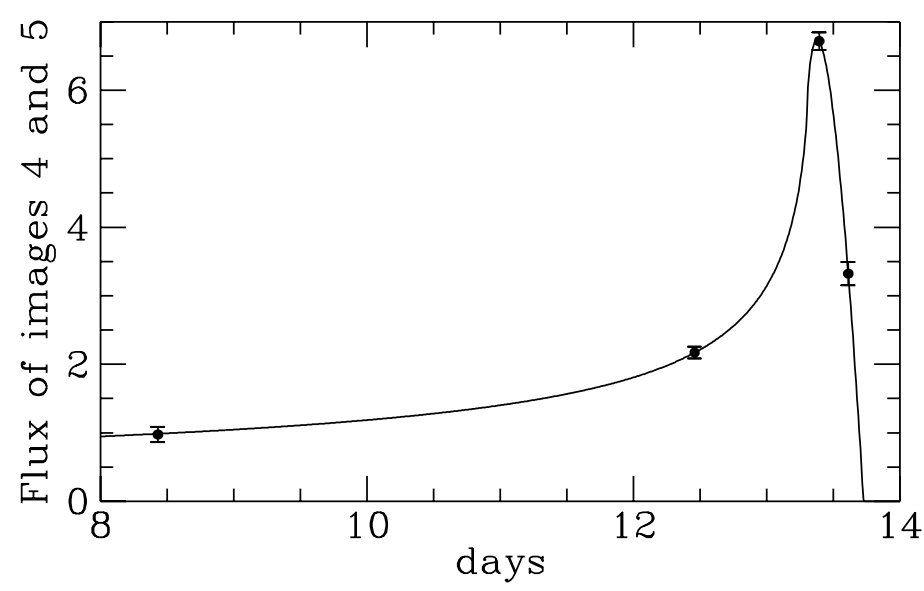

Figure 10. A fit to the 2nd caustic crossing of the binary lens event using the MACHO-Blue band data.

\section{Conclusions}

The microlensing surveys toward the galactic bulge have yielded a number of surprizes including exotic events such as the binary and parallax events. Although they now have the status of curiosities that are interesting in their own right, they also have the virtue that we know more about the properties of the lenses in these events than the lenses in the other microlensing events. With the help of the recently developed "Alert" systems, we will soon be obtaining higher quality data on events that are discovered in progress which should increase the chances of detecting these exotic events. With higher quality data coupled with more observations covering a larger range of $\ell$ and $b$, we hope to shed some light on the major bulge microlensing mystery, which is: Why is the optical depth so high? The resolution of this puzzle will probably help to determine whether the Galactic halo is made predominantly of baryonic or non-baryonic matter.

\section{ACKNOWLEDGEMENTS}

This research was supported by the National Science Foundation through the Center for Particle Astrophysics at UC, Berkeley and by the Department of Energy through the LDRD program at the Lawrence Livermore National Laboratory.

\section{REFERENCES}

Alcock, C., Akerlof, C.W., Allsman, R.A., Axelrod, T.S., Bennett, D.P., Chan, S., Cook, K.H., Freeman, K.C., Griest, K., Marshall, S.L., Park, H.-S., Perlmutter, S., Peterson, B.A., Pratt, M.R., Quinn, P.J., Rodgers, A.W., 
Stubbs, C.W., \& Sutherland, W., 1993, Nature, 365, 621.

Alcock, C., Allsman, R.A., Axelrod, T.S., Bennett, D.P., Cook, K.H., Freeman, K.C., Griest, K., Marshall, S.L., Perlmutter, S., Peterson, B.A., Pratt, M.R., Quinn, P.J., Rodgers, A.W., Stubbs, C.W., \& Sutherland, W., 1994a, in preparation.

Alcock, C., Allsman, R.A., Axelrod, T.S., Bennett, D.P., Cook, K.H., Freeman, K.C., Griest, K., Marshall, S.L., Perlmutter, S., Peterson, B.A., Pratt, M.R., Quinn, P.J., Rodgers, A.W., Stubbs, C.W., \& Sutherland, W., 1994b, ApJ, in press.

Aubourg, E., Bareyre, P., Brehin, S., Gros, M., Lachieze-Rey, M., Laurent, B., Lesquoy, E., Magneville, C., Milsztajn, A., Moscosco, L., Queinnec, F., Rich, J., Spiro, M., Vigroux, L., Zylberajch, S., Ansari, R., Cavalier, F., Moniez, M., Beaulieu, J.-P., Ferlet, R., Grison, Ph., Vidal-Madjar, A., Guibert, J., Moreau, O., Tajahmady, F., Maurice, E., Prevot, L., \& Gry, C., 1993, Nature, 365, 623

Bahcall, J.N. Flynn, C., Gould, A., \& Kirhakos, S., 1994, Nature, in press.

Beaulieu J.P., Ferlet R., Grison Ph., Vidal-Madjar A., Kneib JP., Maurice E., Prevot L., Gry C., Guibert J., Moreau O., Tajahmady F., Aubourg E., Bareyre P., Brehin S., Gros M., Lachieze-Rey M., Laurent B., Lesquoy E., Magneville C., Milsztajn A., Moscoso L., Queinnec F., Rich J., Spiro M., Vigroux L., Zylberajch S., Ansari R., Cavalier F., \& Moniez M., 1994, preprint.

Della Valle, M., 1994 A. \& A ., 287, L31

Giudice, G.F., Mollerach, S., \& Roulet, E., 1993, (preprint: CERN-TH.7127/93)

Gould, A., 1994a, ApJ, 421, L75

Gould, A., 1994b, preprints.

Griest, K., 1991, ApJ, 366, 412

Griest, K., Alcock, C., Axelrod, T.S., Bennett, D.P., Cook, K.H., Freeman, K.C., Park, H.-S., Perlmutter, S., Peterson, B.A., Quinn, P.J., Rodgers, A.W., \& Stubbs, C.W., 1991, ApJ Lett., 372, L79

Kiraga M., and Paczyński, B. 1994, ApJ Lett., in press.

Paczyński, B, 1986, ApJ, 304, 1

Paczyński, B. 1991, ApJ Lett., 371, L63

Paczyński, B., Stanek, K.Z., Udalski, A., Szymanski, M., Kaluzny, J., Kubiak, M., Mateo, M., \& Krzeminski W., 1994 ApJ Lett., 435, L113

Sahu, K.C., 1994, Nature, 370, 275.

Schneider, P., \& Wagoner, R.V., 1987, ApJ, 314, 154

Stubbs, C., Marshall, S.L., Cook, K.H., Hills, R., Noonan, J., Akerlof, C.W., Axelrod, T.S., Bennett, D.P., Dagley, K., Freeman, K.C., Griest, K., Park, H.-S., Perlmutter, S., Peterson, B.A., Quinn, P.J., Rodgers, A.W., Sosin, C., \& Sutherland, W., 1993, SPIE Proceedings, 1900, 192

Udalski, A., Szymanski, M., Kaluzny, J., Kubiak, M., Krzeminski, W., Mateo, M., Preston, G.W., \& Paczynski, B., 1993, Acta Astronomica, 43, 289

Udalski, A., Szymanski, M., Stanek, K.Z., Kaluzny, J., Kubiak, M., Mateo, M., Krzeminski, W., Paczynski, B., \& Venkat, R., 1994a, Acta Astronomica, 44, 165

Udalski, A., Szymanski, M., Kaluzny, J., Kubiak, M., Mateo, M., Krzeminski, W., Paczynski, B., \& Venkat, R., 1994b, Acta Astronomica, 44, 227

Udalski, A., Szymanski, M., S. Mao, R. Di Stefano, J. Kaluzny, M. Kubiak, M. Mateo, \& W. Krzeminski, 1994b, ApJ Lett., in press.

Zhou, H.S., Spergel, D.N. and Rich, R.M., 1994, preprint. 